\title{
Learning to design effective professional development: The influence of integrating a coaching tool with an elementary mathematics specialist course assignment
}

\author{
Courtney K. Baker ${ }^{1}$ (D)
}

Accepted: 27 June 2021 / Published online: 14 July 2021

(c) The Author(s) 2021

\begin{abstract}
As content-specific educational coaches, elementary mathematics specialists (EMSs) have emerged as school-based professionals who are needs-driven and work closely with school stakeholders in regard to mathematics teaching and learning. While leading mathematics education organizations have identified the specialized knowledge and skills required for EMS positions, how to best prepare these individuals is knowledge that the field is still exploring. This paper first presents a theoretical model for EMS preparation that aligns an emerging coaching tool, the Decision-Making Protocol for Mathematics Coaching (Baker \& Knapp, 2019, [DMPMC]) with the Professional Development Design Framework (Loucks-Horsley et al. in Designing professional development for teachers of science and mathematics, Corwin Press, 2010). The paper then presents a descriptive case study that examines the application of this model in an EMS preparation course. The findings indicate that assessing the coaching situation fostered administrative partnerships, revisiting goals increased specificity of anticipated outcomes, and applying research-informed practices increased EMS self-efficacy and advanced coaching agendas. Taken together, these findings suggest that integrating the DMPMC into an EMS preparation course led to positive changes in EMS candidate learning of professional development design. Notably, this is one of the first studies that documents the influence of a coaching education tool on EMS candidates' professional development design.
\end{abstract}

Keywords Elementary mathematics specialist · Professional development - Teacher preparation $\cdot$ Teacher development $\cdot$ Efficacy $\cdot$ Teacher leadership

Courtney K. Baker

cbaker@gmu.edu

1 College of Education and Human Development, George Mason University, 4400 University Drive, MSN 1E, Fairfax, VA 22030, USA 


\section{Introduction}

Elementary mathematics specialists (EMSs) play a critical role in enhancing teacher capacity to enact research-informed teaching practices, implement policy initiatives, and provide professional development to teachers within their school contexts (Association of Mathematics Teacher Educators, 2013 [AMTE]; Fennell, 2017; National Council of Teachers of Mathematics, 2012, [NCTM]). Developing and refining the EMS role in formalized preparation programs is essential as EMSs work with adult learners and have to think about their time and responsibilities differently than when they were in the classroom working only with students. Yet, unlike the broad access of initial teacher preparation programs, only 20 states currently offer an EMS license, certificate, or endorsement and these pathways differ greatly in requirements (Elementary Mathematics Specialists \& Teachers Leaders Project, 2019). As a result of the lack of access to an EMS preparation program, individuals are often "anointed and/or appointed" to EMS positions at both the building and district levels without the requisite content, pedagogical, and leadership knowledge (Fennell, 2017, p. 9). This appointment of non-certified individuals to EMS positions is alarming as targeted preparation is required to facilitate effective professional development experiences that enhance teachers' knowledge of teaching and learning of mathematics (Campbell et al., 2013; Fennell et al., 2013; Showers, 1985). While leading mathematics education organizations have created EMS preparation standards (AMTE, 2013; NCTM, 2012) that forefront the specialized knowledge and skills required to cultivate mathematical knowledge for teaching in others (National Mathematics Advisory Panel, 2008, [NMAP]), how to best prepare EMSs is knowledge the field is still exploring (Campbell \& Malkus, 2014; McGatha \& Rigelman, 2017; Polly et al., 2013; Swars et al., 2018). For example, although a major aspect of their roles as instructional change agents is facilitating professional learning opportunities for school stakeholders, little has explored how EMS candidates' come to their understanding of effective professional development design, facilitation and enactment (Baker et al., 2018; Lesseig et al., 2017)

To address the above-mentioned void, this paper first presents a theoretical model for EMS preparation that aligns an emerging coaching tool, the Decision-Making Protocol for Mathematics Coaching (Baker \& Knapp 2019, [DMPMC]) with the Professional Development Design Framework (Loucks-Horsley et al., 2010). The hypothesis for this theory of action is that the explicit use of the DMPMC not only aligns with the principles of designing effective professional learning experiences but also supports and enhances the teaching of professional development design to EMSs. The paper then presents an empirical descriptive case study that examines the application of this model on EMS candidates. Notably, this is one of the first studies that documents the influence of a coaching education tool on EMS candidates' professional development design. The following research question framed this study: In what ways does integrating a coaching education tool aligned with effective professional development design influence EMS candidates? 


\section{The proposed theoretical model: DMPMC alignment with effective professional development design}

As members of an organizational structure critical for supporting school-wide improvements (Bryk et al., 2010), EMSs present an immediate response to provide the essential expertise required to move the general population of elementary educators forward in enacting high-quality, research-informed mathematics instruction (AMTE, 2013; Campbell \& Malkus, 2011; Fennell et al., 2013; NMAP, 2008). However, EMSs require additional guidance in addressing the content- and context-specific elements of effective professional development design to illuminate research-informed mathematical practices. It is imperative EMSs understand how to design, implement, and evaluate mathematics-specific professional development within their schools that supports systemic reflecting on and learning from practice (AMTE, 2013). To do so, EMSs must determine how to design professional learning experiences that are informed by mathematics-based research (Warfield, 2001), grounded in the mathematics of the student curriculum (Sowder, 2007; Sykes, 1999), and approached through inquiry to emphasize mathematical problem solving and theory building (Lloyd, 2002). EMSs must also address the deep mathematical knowledge that teachers are expected to implement (Ball et al., 2008) along with a clear view of how student mathematical learning progresses across grades (Daro et al., 2011; Sztajn et al., 2012). Furthermore, EMS must apply an understanding of andragogy to their facilitation of professional development so that they can recognize and value the past experiences and knowledge professional development audiences bring to their learning (Darling-Hammond et al., 2017; Trotter, 2006) with regard to mathematics. When these considerations are facilitated effectively, professional development can provide a social situation in which teachers are able to reshape existing beliefs while advancing their mathematical content knowledge and skills (Cooney, 1999).

The proposed theoretical model (see Fig. 1) has the potential to help EMS candidates learn to both negotiate the multifaceted design considerations and facilitate professional

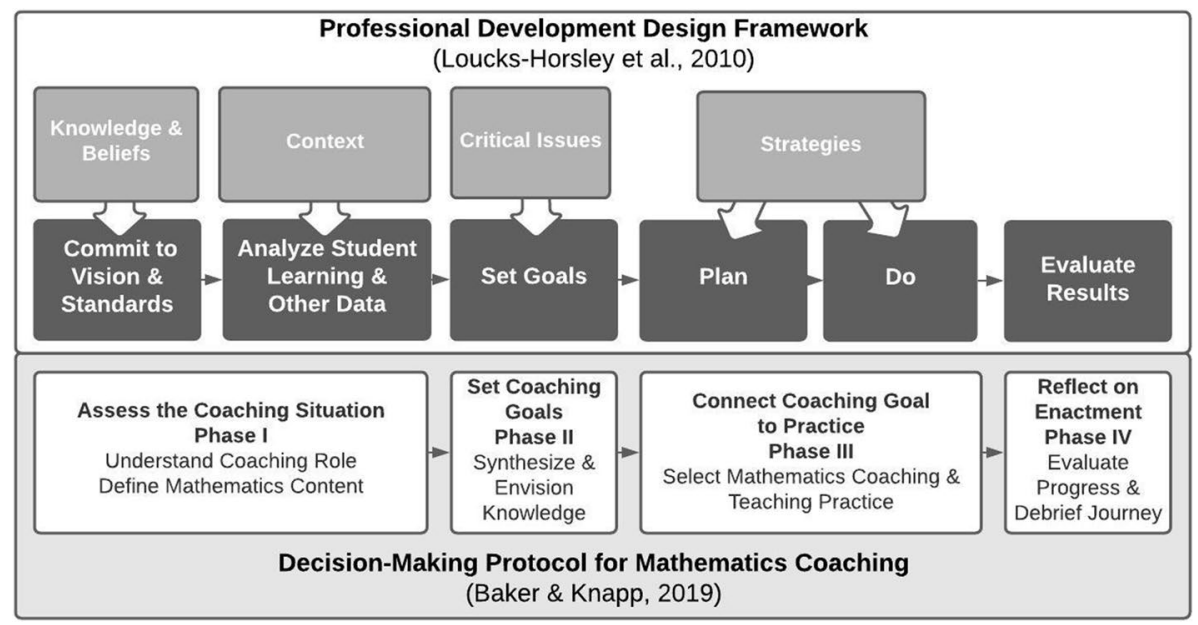

Fig. 1 Proposed theoretical model aligning the Decision-Making Protocol for Mathematics Coaching (Baker \& Knapp, 2019) with the Professional Development Design Framework (Loucks-Horsley et al., 2010) 
learning experiences for transforming teachers' mathematics practice. Aligned with the Professional Development Design Framework (Loucks-Horsley et al., 2010), which conceptualizes considerations of elements required for effective professional development design, the DMPMC's targeted questions have the potential to influence EMS candidates' professional development design. Merging theory and practice, the proposed model takes into consideration influencing factors connected to both teacher and student learning such as knowledge, beliefs, critical issues and strategies (Loucks-Horsley et al., 2010) and brings to the forefront of EMS decision-making school culture, past initiatives, and research-informed teaching (NCTM, 2014) and coaching practices (Gibbons \& Cobb, 2017). Taken together, the targeted questions of the DMPMC can guide EMS candidates through the complex decision-making required to facilitate effective professional learning experiences in mathematics and serve as a reflection mechanism.

\section{The decision-making protocol for mathematics coaching}

The Decision-Making Protocol for Mathematics Coaching (Baker \& Knapp, 2019, [DMPMC]) has recently emerged as a streamlined planning, enactment, and reflection protocol for any mathematics teacher leader, such as an EMS. The DMPMC takes what is known and working for teacher education, integrating teacher practice and research on how people learn mathematics (National Research Council, 2001, [NRC]) to what is known about the design and facilitation of effective professional learning experiences (e.g., Loucks-Horsley et al., 2010). Building on the field of mathematics education's recognition that lesson effectiveness is related to the quality of lesson preparation (Brahier, 2000; Stigler \& Hiebert, 1999), the DMPMC recognizes that the effectiveness of a professional learning experience is related to the quality of implementation preparation.

Designed to guide mathematics teacher leaders in the complex decision-making process of implementing professional learning experiences, the DMPMC serves as a planning and reflection tool that supports EMSs in moving toward the broader goal of improved instruction through the use of core teaching practices (NCTM, 2014). Centered on NCTM's (2014) eight Mathematics Teaching Practices ([MTPs]) and a set of Mathematics Coaching Practices (adapted from Gibbons \& Cobb, 2017; Teachers Development Group, 2010 [MCPs], see "Appendix" the DMPMC challenges people, either individually or collectively, to think critically about their practice and act with intentionality to increase others' mathematics teaching effectiveness. Early evidence of EMSs who utilized the DMPMC has shown increased content-focused interactions and intentionality of their professional learning implementations (Baker \& Knapp, 2019). Additionally, as a coaching tool, the DMPMC provided self-reflection opportunities for EMSs to step out of their personal comfort zone, critically examine their own practice, and target necessary areas of growth in their own practice.

Across all four phases of the DMPMC, the guiding questions prompt individuals to consider both the content- and context-specific issues that are interwoven within each professional learning implementation as a way to leverage a school's culture, differentiate professional learning for school stakeholders, and enhance the learning of teachers to ultimately influence student learning. This is accomplished by situating one's actions on the DMPMC's four phases: Assessing the Coaching Situation (Phase I), Establishing a Coaching Goal (Phase II), Connecting Coaching Goals to Teacher's Practice (Phase III), and Reflecting on Enactment (Phase IV). In the sections that follow, further detail is provided on not 
only each of the four DMPMC phases, but also their alignment with the Loucks-Horsley et al. (2010) Professional Development Design Framework.

\section{Phase I: Assessing the coaching situation}

In Loucks-Horsley and colleagues' (2010) Professional Development Design Framework effective professional development designers must consider not only the context, but the knowledge and beliefs of the audience. Therefore, it is also essential for a facilitator to understand what a particular audience requires and anticipate the possible pitfalls that may occur. The DMPMC Phase I questions prompt users to consider these influences and assess the current school culture, define the mathematics content, and highlight opportunities to improve student learning while simultaneously developing a shared vision for mathematics teaching and learning (see Fig. 2). The DMPMC recognizes that each school setting is a complex organization in which teachers and other school stakeholders are grounded in multiple communities that encompass historical, cultural, political, and social dynamics (Council of Chief State School Officers, 2013). As such, EMSs must analyze and understand not only the affordances and constraints of each context but also the implied cultural norms and explicit regulations and policies (Stein et al., 1999). These school and district policies must be taken into account in order to influence instructional practice (Coburn \& Russell, 2008). Additionally, Phase I assumes students benefit from flexibly moving between multiple representations (Huinker, 2013; NRC, 2009; Stylianou \& Silver, 2004) and assesses the audience's readiness to enact a new instructional practice, as supports for improving teaching and learning are more effective when they are tailored to needs identified by teachers (Darling-Hammond et al., 2017).

\section{Phase II: Establishing a coaching goal}

In their Professional Development Design Framework, Loucks-Horsely and colleagues (2010) prompt professional development designers to consider and address the critical issues that might influence the attainment or influence of professional development goals. Phase II questions of the DMPMC are grounded in strategic goal-setting to build capacity

\begin{tabular}{|c|c|}
\hline $\begin{array}{l}\text { BRIDGING CONTEXT \& COACHING } \\
\text { Understanding the Coaching Role }\end{array}$ & $\begin{array}{l}\text { BRIDGING CONTENT \& TEACHING } \\
\text { Defining the Mathematics Content Focus }\end{array}$ \\
\hline $\begin{array}{l}\text { What are the needs of your audience? } \\
\text { - Are you supporting individual teachers, grade- } \\
\text { level teams, or an entire school? } \\
\text { What is the state of your relationship with } \\
\text { each teacher? } \\
\text { What aspects of the school culture or strategic vision } \\
\text { are essential in your thinking? } \\
\text { - What programs or initiatives have been } \\
\text { implemented or abandoned recently? } \\
\text { - What is the level of receptiveness to coaching? }\end{array}$ & $\begin{array}{l}\text { What is your audience's experience with this } \\
\text { mathematics content? } \\
\text { - What is the current state of teacher } \\
\text { confidence? } \\
\text { - What is the current state of student thinking? } \\
\text { tried? } \\
\text { What resources will support growth in teaching and } \\
\text { learning? } \\
\text { - What representations will promote procedural } \\
\text { - Whency? } \\
\text { What representations will support conceptual } \\
\text { understanding? }\end{array}$ \\
\hline
\end{tabular}

Fig. 2 DMPMC Phase I: Assessing the Coaching Situation (Baker \& Knapp, 2019) 


\begin{tabular}{|l|l|}
\hline $\begin{array}{l}\text { BRIDGING CONTEXT \& COACHING } \\
\text { Synthesizing Situational Knowledge }\end{array}$ & $\begin{array}{l}\text { BRIDGING CONTENT \& TEACHING } \\
\text { Envisioning Changes in Teaching and Learning }\end{array}$ \\
\hline $\begin{array}{l}\text { What connections can you make between the needs of } \\
\text { your audience, the mathematics content, and the goals } \\
\text { of the team/school/district? }\end{array}$ & $\begin{array}{l}\text { What are reasonable and realistic expectations for your } \\
\text { audience? } \\
\text { How will you measure your audience's progress? }\end{array}$ \\
\hline
\end{tabular}

Fig. 3 DMPMC Phase II: Establishing A Coaching Goal (Baker \& Knapp, 2019)

across school stakeholders (see Fig. 3). This phase synthesizes the contextual knowledge gained in Phase I to guide the development of a coaching goal that promotes teacher change by responding to what is known about teacher learning (Gamoran, 2003). Clearly articulated and explicit goals are the foundation of learning (Hiebert et al., 2007), and reasonable and realistic goals can not only motivate learning (Marzano, 2003; McTighe \& Wiggins, 2013), but also guide self-assessment (Clarke et al., 2004; Zimmerman, 2001). Too often, coaching goals are broad and encompass an overall vision for mathematics teaching and learning instead of a targeted outcome. In these instances, it is difficult to determine the effectiveness of a coaching interaction, such as facilitating professional development. To support this, Phase II recognizes both the usefulness of assessment as evidence (NCTM, 2000) and that teachers' beliefs and attitudes will likely only change once increased student learning is observed (Guskey, 1986). Anticipating how one might measure progress in advance of a professional learning implementation provides opportunities to carefully consider what evidence might be collected to highlight both the effectiveness of the interaction in addition to possible next steps.

\section{Phase III: Connecting Coaching Goals to Teachers' Practice}

Once a goal is established, it is then essential to determine the content- and context-specific strategies to best meet the identified goal. In this manner, professional development designers are able to "tailor their program," "consider how to confront challenges," and establish a timeline (Loucks-Hordsley, 2010, p. 41). In the DMPMC, the Phase III questions target the explicit connection of the professional learning experiences' goal to specific coaching

\begin{tabular}{|c|c|}
\hline $\begin{array}{l}\text { BRIDGING CONTEXT \& COACHING } \\
\text { Selecting A Mathematics Coaching Practice (MCP) }\end{array}$ & $\begin{array}{l}\text { BRIDGING CONTENT \& TEACHING } \\
\text { Selecting A Mathematics Teaching Practice (MTP) }\end{array}$ \\
\hline $\begin{array}{l}\text { How will you negotiate and justify the choice of one or } \\
\text { more MCPs? } \\
\text { - Which MCP best aligns with your coaching } \\
\text { situation and your coaching goals? } \\
\text { - What is your readiness to enact the identified } \\
\text { MCP? } \\
\text { - How will this MCP support you in creating a } \\
\text { safe, low stakes learning environment for your } \\
\text { audience? } \\
\text { - What are the obstacles to this enactment that } \\
\text { you will need to overcome? }\end{array}$ & $\begin{array}{l}\text { How will you negotiate and justify the choice of one or } \\
\text { more MTPs? } \\
\text { - If the teachers are unfamiliar with the eight } \\
\text { MTPs, how can you initiate and facilitate a } \\
\text { conversation? } \\
\text { - Which MTPs are most relevant in your } \\
\text { coaching situation? } \\
\text { How will you gauge your audience's readiness to enact } \\
\text { this MTP? }\end{array}$ \\
\hline
\end{tabular}

Fig. 4 DMPMC Phase III: Connecting Coaching Goals to Practice (Baker \& Knapp, 2019) 
and teaching practices. The guiding questions in Phase III (see Fig. 4) prompt reflection upon the context identified in Phase I and the goals set in Phase II assist the EMS determine which MTP and MCP best meets the needs of their audience. It is essential that any shift focused on students' development of mathematical knowledge must be accompanied by a parallel focus on teachers' development of mathematical knowledge (Sowder, 2007). The reflective questions in Phase III also frame the EMS's upcoming work with teachers by situating their actions around two sets of core practices (Grossman et al., 2009): the MTPs which have been identified as those teaching practices most likely to affect student learning (NCTM, 2014) and the MCPs which have been identified as potentially productive coaching activities (Gibbons \& Cobb, 2017). The MTPs and MCPs represent two sets of research-informed practices that support making instructional shifts in school settings.

\section{Phase IV: reflecting on enactment}

Phase IV of the DMPMC is aligned with the Professional Development Design Framework (Loucks-Horsely et al., 2010) in that both highlight the importance of analyzing and reflecting on the resulting evidence from the professional learning experience. Specifically, the aim of Phase IV is a multilayered reflection that centers on the facilitator's practice, the audience's practice, and student learning. Reflection is a powerful tool that can enhance professional development (Sowder, 2007) and encourage teacher change (Schön, 1983). Reflection is a necessity for effective instruction, but rarely occurs without appropriate time or resources (Pugach \& Johnson, 1990). In evaluating professional development, or any coaching interaction, it is essential to reflect on more than the audience's satisfaction (Loucks-Horsely et al., 2010). Additional information is required to determine teacher changes in practice and the influence of these changes on students (Sowder, 2007). The DMPMC's Phase IV questions (see Fig. 5) promote the evaluation of the professional learning experience and the anticipation of future professional learning endeavors to lead to more intentional, proactive conversations that move beyond surface-level interactions toward instructional shifts (Author, 2019).

\section{Methodology}

The purpose of this case study was to understand how integrating the DMPMC (Baker \& Knapp, 2019) with an EMS course assignment influenced EMS candidates' professional development design. The application of an instrumental case study approach was

\begin{tabular}{|l|l|}
\hline $\begin{array}{l}\text { BRIDGING CONTEXT \& COACHING } \\
\text { Evaluating Progress Toward Coaching Goal }\end{array}$ & $\begin{array}{l}\text { BRIDGING CONTENT \& TEACHING } \\
\text { Debriefing and Continuing the Journey }\end{array}$ \\
\hline What evidence will you analyze? & $\begin{array}{l}\text { How will you support your audience in moving forward } \\
\text { on MTPs? } \\
\text { What were your successes? }\end{array}$ \\
$\begin{array}{l}\text { What are your next steps? } \\
\text { How can you improve in your next coaching action? } \\
\end{array}$ & $\begin{array}{l}\text { yourdence will you share with } \\
\text { Whadience so that the mathematics and student } \\
\text { thinking is at the forefront of the } \\
\text { conversation? }\end{array}$ \\
\hline
\end{tabular}

Fig. 5 DMPMC phase IV: reflecting on enactment (Baker \& Knapp, 2019) 
appropriate as the aim of this research was to explore (Yin, 2009), detail and understand the complexities (Stake, 1995) of EMS candidates' interactions with the DMPMC. Recognizing that EMS candidate professional development design experiences are influenced by multiple factors within individual contexts, case study methodology was identified to also help illuminate the decisions and resulting influence of the participants' actions (Yin, 2009). The findings that emerged from this case study will demonstrate if the hypothesized theory of action is correct or if alternative explanations are more relevant.

\section{The elementary mathematics specialist program}

The Mathematics Specialist Leader (K-8) program at George Mason University, established in 2005, provides opportunities for EMS candidates to simultaneously deepen and apply their mathematical content, pedagogy, and leadership knowledge. The program is designed to encapsulate both the expectation of constructed expertise and the range of roles in which the program graduates will engage in as they improve the overall mathematics teaching and learning within their contexts. The 30-credit program meets requirements for state licensure and is aligned with both the AMTE (2013) Standards for Elementary Mathematics Specialists and the NCTM (2012) CAEP Standards for Elementary Mathematics Specialists. The program includes all coursework required for state licensure as a K-8 mathematics specialist in addition to a master's degree in Education Leadership with a concentration in Mathematics Specialist Leadership (K-8). EMS candidates study mathematics content, pedagogy, research, curriculum, and leadership across 10 courses. Each course in the program allows EMS candidates to apply their learning as they promote instructional shifts in practice and advocate for rigorous and coherent learning opportunities within their school settings.

\section{Course context}

The setting of this study was the online synchronous course, Research in Mathematics Teaching. This course was aligned with national EMS standards (AMTE, 2013; NCTM, 2012) and designed to enable EMS candidates to explore and apply research centered on the teaching of mathematics and apply this research to the design of a professional development experience. Since the EMS candidates were practicing educators, it was essential to design course assignments in a manner that flexibly allowed EMS candidates opportunities to demonstrate the development of their mathematics leadership skills authentically. The Professional Development Project, the major course performance-based assessment, required EMS candidates to design, implement and reflect on a professional learning experience of approximately $60 \mathrm{~min}$ for K-12 school stakeholders.

\section{Participants}

The EMS candidates within this course were part of a unique and bounded system (Stake, 2006), defined by enrollment in the Research in Mathematics Teaching course within the EMS preparation program at George Mason University. The 13 EMS candidates meeting the boundaries of this study were experienced educators in a variety of K-12 school positions which included: elementary classroom teachers (5); secondary mathematics teachers (2); school-based EMSs either part- or full-time (4); a district-based EMS (1); and an 
elementary special education teacher (1). All participants, even those positioned as schoolbased or district-based EMSs, were enrolled in a 30-credit graduate program that included coursework for both EMS state licensure and a Master's degree in Education Leadership. Twelve of the candidates were female, and all participants ranged in age from mid-20 s to early $50 \mathrm{~s}$. Candidates represented multiple ethnicities including: White (9), Indian American (2), and Black (2).

A second key criterion for this study included the author as course instructor as multiple sections of this course were offered. Since qualitative research sits squarely at the intersection of researcher and phenomena, it was essential to recognize the influence of the researcher in the observational interpretation of the identified phenomena. In this study, the researcher served as co-developer of the DMPMC, course instructor, and EMS researcher. Integrating the DMPMC within The Professional Development Project provided the researcher with an opportunity to examine and improve EMS learning and explore the efficacy of the DMPMC.

\section{Data collection: Professional Development Project artifacts}

Social constructivism played an essential role within this investigation (Patton, 2002; Shadish, 1995) as the organizational foci (Guba \& Lincoln, 1989) was understanding how the EMS candidates made sense of the DMPMC integration. In addition to researcher memos and reflections, multiple course documents served as artifacts for this study. The timeframe in which these artifacts were collected is depicted in Table 1.

\section{The Professional Development Project documents}

It was essential to examine all documents within The Professional Development Project as the purpose of each of the documents provided opportunities for the participants to communicate their own social construction of the phenomenon being studied. In the Topic Identification and Rationale, EMS candidates were asked to identify and develop a clearly defined focus for their professional development centered on an MTP (NCTM, 2014). Additionally, each candidate provided a rationale for the relevance of their topic to their personal context, other stakeholders within their school and district, and the overall field of mathematics education. The Research-based Conceptual Framework helped EMS candidates to ground their professional developments in research-informed practices. EMS candidates were required to incorporate a minimum of five research articles connected to their identified topic and MTP. Candidates then identified themes and designed a conceptual framework in which they considered the following questions: What does the literature review add to your understanding? What common topics and themes have emerged? What ideas for pedagogical strategies can you adapt?

Similar to a pre-service teacher's preparation of a lesson plan, EMS candidates then created a comprehensive Implementation Plan for their professional development. Essential elements included: timing, materials, clear objectives, detailed activities and actions, planned opportunities for discussion with specific facilitation questions, audience anticipated responses to discussions/questions, and facilitator anticipated responses to audience questions. EMS candidates also integrated considerations for both adult and student learners and included a formative assessment to determine the influence of their professional development and guide future coaching actions. After implementing their professional development, EMS candidates wrote a Final Reflection Paper in which they reflected on 


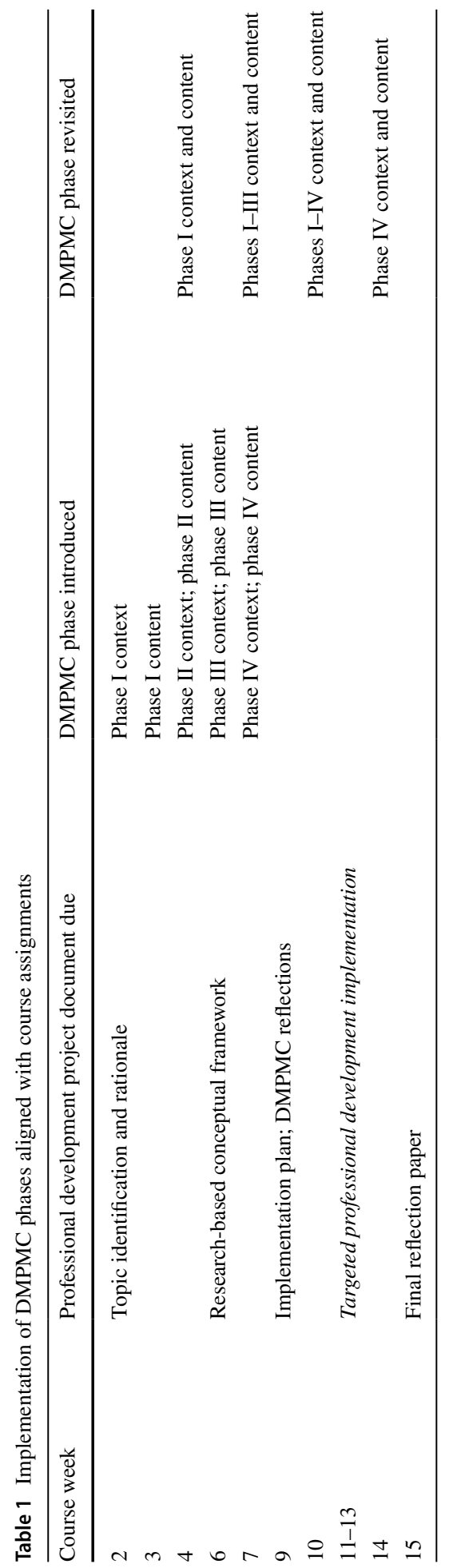


the following: the role of learning and teaching of mathematics, the role of mathematics instructional leaders, the improvement of student learning, and continuing the implementation within their contexts.

\section{DMPMC Reflections}

The DMPMC was integrated throughout each stage of the Professional Development Project. Doing so, the DMPMC served as a reflective framework at each stage targeted at supporting EMS candidates in the following ways: differentiating their professional development, meeting the specific needs of school stakeholders, and considering the complexities of designing and delivering professional development. Throughout the semester EMS candidates were asked to apply the knowledge gained from the DMPMC's reflective questions to support their anticipation of teacher responses and implementation of their identified MTP and MCP. The DMPMC questions were parceled out during the first half of the course to provide EMS candidates with time to intentionally reflect on each subset of questions as they pertained to the professional development design. Each phase of the DMPMC was introduced in isolation between weeks 2 and 7 (see Table 1). During weeks 5 through 7 EMS candidates explored the potentially productive coaching activities (Gibbons \& Cobb, 2017) that the MCPs are centered on via class readings and discussions. During weeks 7 through 13 EMS candidates revisited and re-reflected on the DMPMC guiding questions in Phases I-IV as they designed and prepared for their professional development implementation. EMS candidates also revisited the DMPMC questions before the final submission of the Professional. Development Project assignment in week 14. This intentional revisiting of the DMPMC reflective questions was conducted in parallel with the Professional Development Project assignment and afforded the EMS candidates opportunities to add to or revise their initial professional development design. Due to the ongoing nature of this EMS course assignment, instructor feedback was provided throughout the semester, often in the form of questions. Examples of these prompting questions are: How might your audience respond? What questions might you use to facilitate this discussion?

\section{Data Analysis}

A qualitative approach was applied to the course artifacts so that the EMS candidates' perceptions and resulting interactions could be accurately reported (Shadish, 1995) and give voice to participant experiences (Weiss \& Greene, 1992) to gain an experiential understanding of the case (Stake, 2006). An in-depth exploration of the phenomenon (Patton, 2002) was conducted by first chronologically analyzing each Professional Development Project document and DMPMC reflections across participants. Examining the codes as they emerged across the semester captured the uniqueness of each case (Patton, 2002) to be recognized over time. An InVivo coding scheme was applied to capture the specific terms used by the candidates (Saldaña, 2016). Each course artifact underwent analysis in which excerpts, passages, and open-ended responses to reflective prompts were studied. Values coding (Saldaña, 2016) served as a secondary coding technique that captured not only the participants' values, but also their attitudes and beliefs. Analytic memos were written to allow for researcher reflexivity and a critical analysis of the phenomenon (Saldaña, 2016). Although some codes were anticipated in advance (e.g., school/district goals, teacher learning, confidence), other codes emerged during analysis (e.g., administrative support, value of research-informed practice). Codes were organized around broad 
topics and substantive categories (Maxwell, 2005) and afterwards compiled on a master list and reviewed for redundancy. Codes that were conceptually similar to one another were grouped together in categories (Saldaña, 2016) and labeled with major headings (e.g., goals, feedback and value, facilitation of professional development, perceived belief of audience). Analysis across categories included the examination of patterns in the candidate's role and their positioning of school stakeholders.

To develop themes, initial codes and categories were aligned with the four phases of the DMPMC. Categories were then re-aligned with the originating documents to determine underlying elements of the project that may have contributed more strongly to candidate thinking. A matrix was created to organize the examination of common categories across participants (Patton, 2002), and evidence was placed within each category (Yin, 2009). Categories were subsequently grouped together which resulted in the emergence of themes. In order to develop a clear understanding of the DMPMC influence on the planning and implementation of participant professional development, evidence to support and/or refute each theme was pulled from course artifacts.

The multiple examinations of each document increased the validity and established the trustworthiness of the identified findings as the assignment documents were submitted at various points during the 15 -week class. Furthermore, the author consulted with the codesigner of the DMPMC throughout the study implementation and analysis. These conversations provided alternative perspectives from a source familiar with the DMPMC, professional development design, and EMS development.

\section{Findings}

This study sought to understand how integrating a coaching education tool aligned with an effective professional development design framework (Loucks-Horsley et al., 2010) influenced EMS candidates' professional development design throughout the course of one semester. Analysis of the EMS candidates' Professional Development Projects revealed the following findings: (1) Assessing the coaching situation fostered administrative partnerships; (2) Revisiting goals increased specificity of anticipated outcomes; and (3) Applying research-informed practices increased self-efficacy and advanced coaching agendas. Taken together, these findings suggest that engaging with the DMPMC helped prepare EMS candidates to design professional development.

\section{Assessing the coaching situation fostered administrative partnerships}

The guiding questions in Phase I of the DMPMC allowed the formation of new partnerships, expanding the candidates' perspectives to the broader school community context. Each of the DMPMC's Phase I questions encouraged EMS candidates to critically examine the context of their upcoming professional development by reflecting on the school's culture and strategic vision. Due to many of the candidates' positioning as classroom teachers, conversations with administrators were essential to answer the Phase I questions and gain the greater context of their school communities. Many EMS candidates positioned as classroom teachers had not yet thought broadly about their school's mathematics programs. Instead, they were appropriately focused on the mathematics teaching and learning most relevant to their employment. Now that they were being asked 
to design professional development for extended audiences, EMS candidates required additional information to meet their audiences' needs.

I want to consult with my administration and math resource teacher because I am not completely sure how to fully answer the DMPMC questions. I have very little interactions with the teachers in my school outside of the grades I work with (Hazel, DMPMC, Phase I Reflection).

During conversations with administrators, EMS candidates gained valuable insight into the overall school context. In some instances, candidates gained a longitudinal perspective of their school's needs and were able to envision an alignment with their own potential contributions.

I am new to the school and not familiar with programs and initiatives from past years. This year the district has been promoting and expecting the implementation of the math workshop model. There has also been a big push through professional development for teachers to implement Number Talks. I see this as one option for an opening routine in the workshop model (Lily, DMPMC Phase I Reflection).

After their initial conversations, EMS candidates believed the administrators viewed them as individuals who possessed specialized knowledge on making instructional shifts. They shared that their administrators were "open to feedback" and "valued [their] perspective." In several instances, administrators encouraged candidates to share their professional development with "others in the building," thus empowering and fostering the leadership of the EMS candidates. This afforded opportunities for the candidates to view their administrators as collaborative partners in developing their school's teaching force. For example, many administrators assisted the EMS candidates with designing their professional developments.

My [primary] supervisor reviewed my activity and gave me suggestions on how to structure questioning while the building principal enthusiastically promoted the activity with her teachers and provided the physical space. Their leadership showed a dedication to improving math instruction and commitment to trying new things. I truly am thankful for having math leaders that I feel safe to ask questions of and learn from. (Julia, Final Reflection Paper).

I worked with my administration to revise and finalize my plans for the training session and was pleasantly surprised by the positive and encouraging feedback they provided before the delivery date. They were very supportive of my reasoning for choosing this topic and felt it perfectly aligned with our School Improvement Plan. The feedback from my administrators allowed me to walk into my professional development with a level of confidence and excitement because of my passion surrounding this topic and the effort I put into research and planning (Hazel, Final Reflection Paper).

EMS candidates often extended these new partnerships by inviting administrators to attend their professional developments. In this manner, candidates advocated not only for themselves and future leadership roles, but also their visions of mathematics teaching and learning. This advocacy led to the unveiling of new leadership possibilities for the EMS candidates.

Looking to the future, my principal has mentioned that we are taking on a number of new staff members next year and would like me to prepare some professional 
development specifically for them at the beginning of the year when teachers are setting routines and procedures (Natalie, Final Reflection Paper).

The application of this specialized leadership knowledge afforded EMS candidates the opportunity to explore new positionalities within their schools. Subsequently, the combined knowledge gained from administrative collaborations promoted the transition of EMS candidates' identities from teachers advocating for mathematics within their classrooms to leaders advocating for mathematics throughout their schools.

\section{Revisiting goals increased specificity of anticipated outcomes}

EMS candidates answered and revisited their responses to the DMPMC's guiding questions throughout the first ten weeks of the course. Initially when reflecting on the Phase I questions in Weeks 2 and 3 candidates' goals centered on "providing information" and "supporting teachers" through exploration of their identified MTP.

I feel that the research on purposeful questions will be an important resource. I also think modeling what it might look and sound like will be important. If teachers can identify what type of questions provide the most information about student learning, and provide the most opportunities for a student to make connections, that might be a useful tool (Sophia, DMPMC Phase I Reflection).

These preliminary goals emphasized a desire for the professional development audiences to experience the learning of mathematics similar to the EMS candidates' program experiences.

The audience will experience three act tasks through the lens of a student like I did. Experiencing what "productive struggle" feels and looks like will help teachers better scaffold tasks in their own classrooms. After experiencing what three-act tasks "feel like" teachers will reflect in small groups on classroom vignettes from several articles (Julia, DMPMC Phase I Reflection).

I want teachers to make a connection between conceptual and procedural understanding as students learn and practice their math facts. I think for this to happen, teachers need to see that there are multiple ways to approach multiplication and division even when the fact is not known. I want to encourage a variety of strategies and have teachers model their thinking just like they would hopefully want their students to do (Natalie, DMPMC Phase I Reflection).

When candidates reflected on the DMPMC Phase II questions in Week 4, they further refined their goals. As candidates synthesized the situational knowledge and envisioned changes in their audiences' practice, EMS candidates' goals transitioned from audiences receiving resources to audiences "immediately implementing" resources into classroom practice.

Since my professional development is to build procedural fluency from conceptual understanding using a math workshop model, I am hoping that my audience will be able to incorporate either math workshop or station ideas into some of their lessons as a way to help promote the building of procedural fluency from conceptual understanding. This will give students challenging math tasks that they can work on in small groups or stations (Jessie, DMPMC Phase II Reflection). 
In revisiting their goal-setting in Week 6, EMS candidates often further enhanced their goals to include their audiences developing an increased understanding of a researchinformed MTP.

I want to share with my colleagues the importance of providing students with tasks that involve high-level cognitive demands. We will explore a bit about what research says students needed, how to determine if a task is low-level or high-level demand, maintaining high-level demand, and creating their own tasks as a way to connect to what was covered in the PD and a takeaway after the PD (MacKenzie, DMPMC Phase III Reflection).

As EMS candidates gained specificity in their goal-setting, a parallel shift in the proactiveness of their outcomes emerged. EMS candidates' initially anticipated follow-up actions were vague and minimized the EMS candidate's potential influence by positioning themselves as passive providers of resources such as video links, emails and flyers. EMS candidates also hedged their influence by using phrases like "I hope to," "if the teachers want," and "make myself available." Revisiting the Phase IV questions throughout the semester allowed EMS candidates to advance their anticipated outcomes and express proactive follow-up actions including: meeting with individuals and teams of teachers, inviting other school stakeholders to observe their teaching, creating resources that targeted the audience's needs, and scheduling demonstrations.

For instance, when answering the Phase IV questions in Week 6 the follow-up actions that Amber, a K-6 EMS, anticipated were centered on interactions at "some point" in the future. "In my next coaching action, I hope to be able to engage teachers with the mathematics after planning goals for the lesson and assure that incorporated tasks are at a high level of thinking and reasoning." However, in Amber's final reflection her follow-up actions were more targeted. She discussed planning upcoming lessons with her audience and regularly attending team meetings. Additionally, she spoke to developing an assessment tool to guide her future coaching interactions.

Beginning next week, I will be meeting with teachers to follow up on specific questions and planning for opportunities to support their next steps. I have the opportunity to meet with individuals to support them and to also meet during their gradelevel professional learning community meetings. First grade teachers have asked me to meet with them to incorporate more opportunities for formative assessment during math workshop. We will work together to plan upcoming mini-lessons to make sure mathematics goals are aligned and are clear. Then, we will look to see where it makes sense to use formative assessment in order to inform their instruction. Each teacher has unique questions and requests for next steps and I will carefully consider whether we will meet one-on-one or in grade-level groups (Amber, Final Reflection Paper).

As an individual already employed as an EMS, Amber was already positioned to collaborate with teachers during their team meetings. However, it was not until she revisited the Phase IV questions that Amber provided evidence of using her role to actively engage her audience in an instructional shift.

EMS candidates' positions within their school played a central part in identifying their initial follow-up actions. Those who were classroom teachers anticipated fewer opportunities in Week 6 to interact with the audience due to their positioning and described passive actions. "I will plan to make the Math Teaching Practices visible through shared school bulletin boards and flyers sent out for teachers to have hanging in their classrooms to refer 
to during math instructional blocks" (Hazel). However, in Week 14 Hazel re-imagined how she could use her positioning as a classroom teacher to her advantage and support other school stakeholders.

One follow-up idea I have is to record myself in the classroom implementing a highlevel task with my students. I would like to focus on different parts of implementing tasks to also highlight my chosen Math Teaching Practice clearly demonstrated at each point of implementation (Hazel, Final Reflection Paper).

These changes in leadership and advocacy were also visible in other EMS candidates' DMPMC reflections. Reya, a classroom teacher, initially wanted to survey the audience for her follow-up actions. By the time she implemented her professional development she planned a follow-up meeting to "work together with other teachers to implement the space station task in the classroom." Jessie, another candidate, positioned as a classroom teacher, initially anticipated in Phase IV that he would "try to start conversations." When he revisited the Phase IV questions, his follow-up actions included more than simply attempting conversations.

It is my plan to follow-up with each teacher regardless of whether or not I get feedback. My goal is to meet briefly with each individual teacher to gauge their comfort level with implementing the math workshop and other ideas put forth in this PD (Jessie, Final Reflection Paper).

Responding to the DMPMC questions multiple times throughout the semester prompted EMS candidates to advance their thinking on follow-up actions that actively engaged their audiences in instructional shifts. Although many EMS candidates were initially unable to envision these actions, as they revisited the Phase IV questions the specificity of their future interactions increased. This empowered candidates and allowed them to view themselves as instructional change agents; ultimately providing the EMS candidates with a heightened awareness of providing influential professional development centered on actions that promote teacher change, a primary role of an EMS.

\section{Applying research-informed practices increased self-efficacy and advanced coaching agendas}

EMS candidates were quick to identify the instructional changes of mathematics teaching and learning they desired within their schools as they grounded the design of their professional development in research-informed practices. However, when anticipating audience responses, the EMS candidates expressed a lack of confidence. Candidates perceived that their audiences, primarily composed of their peers, would not value the professional development. Many candidates worried that they "lacked the experience" required or were "not established enough" for their audiences to have buy-in, and expressed these thoughts as obstacles in their DMPMC reflections.

This is only my second year in this district so I am still getting to know my colleagues and administration. Being such a new face, I am concerned with how others will accept what I have to share. They have expressed apprehension to new ideas and approaches (Miranda, DMPMC Phase I Reflection). 
These teachers are a little more set in their ways and it is harder to get them on board to the changes in curriculum or the way we could teach a lesson. Depending on the topic, they may be more open to changes and trying something new than other times (Natalie, DMPMC Phase I Reflection).

A second obstacle perceived by the EMS candidates was the timing of the professional development implementation aligning with the district standardized assessment testing windows. EMS candidates believed that their audiences would be solely focused on the upcoming summative assessments and lack readiness for learning about research-informed practices that "could not immediately be used."

Time is always a factor for teachers. While teachers might see the value of Three-Act Tasks, they are focused on the [state standardized assessment] review at this time of year and might not see how they can implement Three-Act Tasks while reviewing effectively. There might also be hesitance with trying something new (Julia, DMPMC Phase II Reflection).

Despite EMS candidates' anticipation of multiple obstacles, reality differed. After facilitating their professional development, candidates were surprised at the audience engagement as many school stakeholders expressed a willingness to step out of their comfort zone.

Even though I had reservations, my professional development was well received and I had some great feedback. One teacher said, 'This made me realize I need to step away from the textbook and work in more ways for students to use the skill in a task. Tasks are more fun and worthwhile!' Having this response from this, one teacher made the entire professional development worth it for me (Miranda, Final Reflection Paper).

The EMS candidates' audiences valued the insight and knowledge provided within the professional developments. Specifically, in their final reflections, EMS candidates cited that their audiences "enjoyed learning about the research" and "engaging in rich mathematical thinking." This was "the biggest takeaway" for the EMS candidates.

All of the teachers remarked on how powerful it was to see the different ways of approaching the problem, and it helped to create buy-in for why higher-level tasks are so important for our students. They felt the task would really stretch and push student thinking (Reya, Final Reflection Paper).

In their final reflections, candidates also spoke of the individuals and teams from their professional development audiences that requested "follow-up tasks" and "more professional development" on the research-informed MTPs. EMS candidates were encouraged that others "acknowledged [their] leadership skills" and "were impressed by [their] level of expertise."

However, it was not only EMS candidates' perceptions of their audience that increased positively after implementing the professional development. When school stakeholders scheduled coaching interactions or shared thoughts on future implementations of the ideas gained, EMS candidates expressed an increase in their own confidence and perceived value. This increase in self-efficacy empowered the EMS candidates.

More than half of the teachers wrote they would continue the structure I shared within their math workshop. One teacher said she wanted to have a question on the menu, with each activity, for students to answer. Another teacher mentioned that she was going to begin writing questions on the workshop menu. This helped me to see 
that people really need support to integrate rich math questioning. Moving forward, each week at our math meeting I will start with a task and collaborate with the team to create one question that might further students' thinking or provide an opportunity for them to make a connection (Sophia, Final Reflection Paper).

Upon leading my first Professional Development on my own, I now feel more confident that this is an area I feel a calling to and passionate about. I know teachers want the time to invest in learning new teaching practices that will benefit their students and see a need for our instructional practices taking students beyond passing a highstakes exam. Teachers reflected that they see a need for implementing these highlevel tasks so students are able to not only access content and context of problems, but are also led to think deeply (Hazel, Final Reflection Paper).

Across all phases, the guiding questions of the DMPMC emphasized the need for professional development facilitators to consider the broader context and focus on the research-informed practices most applicable to their context. Taking this information into account allowed EMS candidates to scaffold their professional development to meet their diverse audience needs and ultimately afforded the candidates opportunities to grow as mathematics leaders.

This experience has helped me grow as an emerging teacher leader. At first, I was very nervous and somewhat unsure of myself as I led the PD. As I got into the session and started to interact with the audience, I began to feel more comfortable about my role as facilitator. I sensed that I was being more effective in this role as my audience really began to engage in discussions of mathematics teaching and learning. This experience of creating and implementing a professional development session has been an invaluable episode for me (Jessie, Final Reflection Paper).

\section{Discussion}

This aim of this study was to understand how integrating a coaching education tool aligned with effective professional development design influenced EMS candidates. The choices EMS candidates make about which aspects of school culture and practice to apply in their designed learning experiences are important work that must not be taken lightly. As an EMS candidate determines what teaching and coaching practices to enact and which aspects of instruction to focus on, bringing their decision-making to the forefront can promote reflection on how their actions may be helping or hindering teacher change within their schools.

The findings indicate that EMS candidates' efficacy on designing and delivering professional development increased as a result of intentional planning and reflection. This follows logically, as over half of the EMS candidates were positioned as classroom teachers and this was their first attempt at facilitating a professional learning experience. However, the findings of this study further indicate that considering the DMPMC's guiding questions multiple times throughout the design of the professional developments led to an increase in specificity and proactiveness in EMS candidates' goals and outcomes. This is notable as professional development designers must understand how to set and evaluate goals that support teacher change and influence student outcomes (Campbell \& Malkus, 2011; 
Guskey, 2000). The DMPMC purposefully guided EMS candidates in determining and refining a goal for this coaching interaction; ultimately moving both the candidate and their audience forward in their respective practice. By setting attainable goals (Phase II), evaluating their effectiveness (Phase IV) and anticipating the next coaching interaction (Phase IV) before the professional development implementation, EMS candidates envisioned their leadership roles beyond this single professional development experience. This cycle of creating and then analyzing goals while simultaneously visualizing future coaching interactions was strategic and supported EMS candidates in this study with not only developing a proactive practice but also envisioning themselves as leaders.

Initially, many EMS candidates in this study perceived their audiences as hesitant or unreceptive. Shifting EMS candidates away from these unproductive perspectives in EMS preparation programs is especially important as beliefs guide practice (Beswick, 2012). As leaders within their schools, EMSs must build communities of reflective teachers and provide professional development for teachers to acquire new skills and strategies for their pedagogy (Campbell et al., 2013; Fennell et al., 2013; Showers, 1985). Thus, it is essential that EMS think positively of not only their own influence, but also the school stakeholders with whom they work. In this study, the DMPMC pushed EMS candidates to investigate and understand other school stakeholders' perspectives so that the professional development design could build on teachers' work already in place. Similar to how teachers must actively envision the approaches their students take when working on a mathematics task (Lampert, 2001; Schoenfeld, 1998; Smith, 1996; Stigler \& Hiebert, 1999), EMS must consider how teachers and other school stakeholders might interpret and implement each professional learning experience. Understanding and addressing the audience perspective is essential as every teacher's work makes sense from their own perspective (Simon \& Tzur, 1999) and sustained change results from professional development experiences that connect change in the audiences' beliefs and practice (Phillips, 2007). Within this study, EMS candidates used the context-centered questions across all phases of the DMPMC to consider the ways in which they could connect audience needs (Phases I, II, III) with the mathematics practices they advocated for (Phase III), thus anticipating audience hesitations (Phases I, II, III). If EMS candidates understand and integrate the professional culture of a school into their work, widespread shifts in both practice and school culture can then occur (Gamoran et al., 2003; Sowder, 2007).

School-centered instructional shifts also require administrators and EMSs to collaboratively create spaces within existing school structures (Hjalmarson, 2017; Knapp, 2017) so that a shared vision for leadership can be realized (Chval et al., 2010) and distributed throughout the school community (Gamoran et al., 2003). In this study, when EMS candidates sought answers to the DMPMC's guiding questions from their administrators a partnership was formed. These partnerships played a critical role in not only the design of EMS candidates' professional development, but also the fostering of EMS candidates' leadership knowledge and skills. For example, many candidates initially hedged their anticipated influence and planned for small coaching interactions with grade-level teams. Upon hearing this, their administrators helped guide the EMS candidates to see the greater potential in their professional development and pushed them toward larger audiences. This action demonstrates how the administrators saw the EMS as partners in making instructional shifts within the school, which ultimately empowered the EMS candidates to further this identity. After these collaborations, many EMS candidates viewed administrators as collaborative partners in advocating for both the mathematics practices they espoused and their potential leadership roles. Specifically, this fostered the creation of a shared vision for the 
EMS candidate and administrator. Administrative support is essential as school and district policies influence both access to instructional supports and the allocation and identification of resources required to make instructional shifts (Coburn \& Russell, 2008; Sowder, 2007). EMS candidates must learn to advocate for and educate others on research-informed practices for mathematics teaching and learning as many school stakeholders, including administrators, still view learning mathematics as procedural and hierarchical (Nelson, 1998). By engaging in advocacy of research-informed mathematics teaching and learning with their administrators, EMS candidates gained specialized leadership knowledge (Sowder, 2007), confidence, and an understanding of a principal's influence on school-initiatives and professional development (Coburn \& Russell, 2008).

Ultimately, integrating the DMPMC with an EMS course assignment served as a catalyst for EMS candidate development; encouraging an understanding of and alignment with research-informed practices to support the teaching and learning of mathematics in ways that research suggests is effective. Similar to pre-service teachers learning to write and reflect on their lesson plans, this course assignment provided EMS candidates with explicit opportunities to write and reflect on their professional development design. Planning is not only a skill that can be learned, but also one that can greatly influence the quality of instruction (Stigler \& Hiebert, 1999). With regard to EMS candidate preparation, it is the purposeful planning that includes the creation of targeted goals and objectives, as well as the inclusion of anticipated audience responses, that will foster EMS candidate growth and prepare these individuals to act as agents of change. In this study, revisiting the DMPMC's guiding questions prompted EMS candidates to narrow their focus, identify data sources to determine influence, and immediately consider future actions for advancing instructional shifts.

\section{Implications}

In their development of the DMPMC, Baker \& Knapp (2019) presents the notion that with intentional planning and reflection, the practice of mathematics teacher leadership can be learned and continuously improved. They further suggest the integration of the DMPMC with EMS candidate course assignments to "build understanding of preparing and facilitating professional development for practicing teachers" (p. 36). In this study, the DMPMC was a guiding influence in leveraging an EMS course assignment centered on effective professional development design and delivery. Based on the findings, EMS candidates fostered administrative partnerships, increased goal specificity of anticipated outcomes, and experienced an increase in self-efficacy for providing professional development. Taken together, these findings suggest that integrating the DMPMC with a professional learning experience led to positive changes in EMS candidate learning of professional development design. Additionally, these findings indicate that the EMS candidates advanced their understanding of the various contextual influences within the broader school community and advanced collaborations with many school stakeholders.

This study contributes to the void in EMS literature by proposing a model to support EMS professional development design and offering insight into an EMS course assignment integrated with a coaching tool. It is known that an ongoing challenge with EMS preparation is that coaching applications vary widely (Poglinco et al., 2003; Resnick, 2010) and EMS candidates are already employed as school practitioners. However, this EMS course assignment allowed all EMS candidates no matter the grade-band, experience or position 
to engage in and refine essential leadership skills. This research also has the potential to inform and influence other EMS preparation programs in their design of EMS course assignments aimed at developing the specialized knowledge required for EMS candidates to facilitate high-quality professional development (AMTE, 2013; NCTM, 2012). As EMS mathematics teacher educators share their work, the knowledge base of how to best prepare EMSs will expand. Furthermore, integrating the DMPMC with course assignments offers a structure that EMS candidates can return to beyond their initial preparation programs.

Future iterations of this assignment will be assigned more strategically as instructors will have the ability to highlight the way in which this particular assignment enhanced EMS candidate knowledge and skills. For instance, mathematics teacher educators can emphasize to EMS candidates that revisiting the DMPMC questions multiple times throughout the duration of a semester was particularly salient by affording opportunities for EMS candidates to dig deeper into their decision-making and reflect on their actions to ultimately promote instructional shifts within their school settings. The EMS candidates in this study were able to switch between the DMPMC's four phases over a period of 15 weeks, indicating that there is not necessarily one path to increase intentionality as seen in past research (Baker \& Knapp, 2019). Instead, it is the idea of going back and reflecting on one's practice that made the integration of this tool more influential in EMS candidate professional development design and delivery.

It is also notable that the EMS candidates within this study primarily identified the MTPs and MCPs that they had seen explicitly modeled in their EMS coursework. This indicates that EMS candidates utilized this EMS course assignment to not only demonstrate their new knowledge, but also develop professional learning presentations aligned with their instructors' modeled practices. While this provides evidence that the EMS candidates intentionally applied the MTP and MCPs they had seen explicitly modeled in their coursework, this also highlights the importance of needing to include a variety of experiences with the MTPs and MCPs embedded throughout EMS preparation programs. In this manner, EMS candidates will have opportunities to revisit and reexamine their practice under the supervision of a mathematics teacher educator who can guide and support their reflective practice. This will also both heighten EMS candidate awareness of and increase their experiences with all MTPs and MCPs: practices that may be unfamiliar or uncomfortable.

This study was designed to examine EMS candidate reflections across a single semester. It is recognized that additional research is required to determine how EMS candidate reflections on their Professional Development Projects are aligned with school stakeholders' perceptions of the professional development implementations. There is also a need to closely examine the partnerships between EMS candidates and administrators. If the ultimate goal is to influence mathematics teaching and learning so that all students are supported, these partnerships must continue to center on established common goals and expectations. Future research should build on how these partnerships between EMS candidates and administrators are formed and the influence these partnerships have over time across other school stakeholders. Additional research is also required to further explore the development of EMS candidate goal-setting. What types of goals do EMS candidates make and how is progress for those goals measured along the way? Observations of EMS candidates engaging in their work within their school communities will help achieve these aims by better connecting EMS beliefs and actions, in addition to driving future research. Additional research that examines the influence of the EMS candidate on other school stakeholders will provide necessary insight into the preparation of teachers as EMS (Swars et al., 2018). 
EMSs have the potential to positively influence instruction and learning within preK12 schools (Campbell \& Maulkus, 2011). It is important that advanced certification programs for practicing teachers, such as EMS certification programs, offer opportunities for candidates to connect learning to practice while reflecting deeply on practice. Integrating coaching tools that enhance professional development design, such as the DMPMC, has the potential to support EMS candidates' acquisition of the necessary content, pedagogy, and leadership skills for becoming school-based leaders. If mathematics teacher educators can develop effective advanced program coursework that prepares EMS for leadership positions within school communities, there exists the potential to influence not only the individual EMS candidate's knowledge and understanding, but the collective knowledge of all school stakeholders.

\section{Appendix}

\footnotetext{
Engage in Mathematics Effective coaching of mathematics encourages collaborative discussions and problem solving to plan lessons, build content knowledge, anticipate or analyze student responses, prepare purposeful questions, explore manipulatives, select mathematics goals, and analyze the rigor and quality of the mathematics tasks. Engaging in mathematics within these activities deepens teachers' specialized disciplinary knowledge

Examine Student Work Effective coaching of mathematics facilitates collaborative conversations centered on examining student work samples to identify student understanding and misconceptions, to develop a shared understanding of student conceptualization of a mathematics topic, and to inform the next instructional steps

Analyze Classroom Video Effective coaching of mathematics guides conversations on shared teaching experiences to reflect on aspects of teaching. Videos can serve as representations that support teachers in learning and refining their practice
}

Rehearse Aspects of Practice Effective coaching of mathematics orchestrates pre-planning of specific practices and conversations to provide opportunities to practice aspects of teaching and provide and receive feedback

Engage in Lesson Study or Studio Day Effective coaching of mathematics brings together groups of teachers, administrators, and/or instructional specialists in a collaborative professional development. One of more cycles of lesson study or studio day may include planning a lesson, observing the implemented lesson, gathering student evidence, analyzing student data, and reflecting on the enactment and outcomes. These forms of professional development also provide opportunities for coaches to help teachers deepen their content knowledge

Co-teach Effective coaching of mathematics involves supporting teachers with delivery of instruction in the classroom. The coach and teacher work collaboratively to purposefully plan interactions to maximize student learning and enact particular practices to improve instruction

Model Instruction Effective coaching of mathematics involves demonstrating for teachers the delivery of instruction in the classroom. The coach and teacher work collaboratively to purposefully highlight coach-student interactions to maximize student learning

Mathematics Teaching Practices. Adapted from Author, 2019; Gibbons \& Cobb, 2017; Teachers Development Group, 2010.

Author contribution Not applicable

Funding Not applicable 
Availability of data and material Because participants may be identified based on their qualitative data, these will not be made publicly available.

\section{Declarations}

Conflict of interest Not applicable

Code availablity Upon request

Open Access This article is licensed under a Creative Commons Attribution 4.0 International License, which permits use, sharing, adaptation, distribution and reproduction in any medium or format, as long as you give appropriate credit to the original author(s) and the source, provide a link to the Creative Commons licence, and indicate if changes were made. The images or other third party material in this article are included in the article's Creative Commons licence, unless indicated otherwise in a credit line to the material. If material is not included in the article's Creative Commons licence and your intended use is not permitted by statutory regulation or exceeds the permitted use, you will need to obtain permission directly from the copyright holder. To view a copy of this licence, visit http://creativecommons.org/licenses/by/4.0/.

Open Access This article is licensed under a Creative Commons Attribution 4.0 International License, which permits use, sharing, adaptation, distribution and reproduction in any medium or format, as long as you give appropriate credit to the original author(s) and the source, provide a link to the Creative Commons licence, and indicate if changes were made. The images or other third party material in this article are included in the article's Creative Commons licence, unless indicated otherwise in a credit line to the material. If material is not included in the article's Creative Commons licence and your intended use is not permitted by statutory regulation or exceeds the permitted use, you will need to obtain permission directly from the copyright holder. To view a copy of this licence, visit http://creativecommons.org/licenses/by/4.0/.

\section{References}

Association of Mathematics Teacher Educators. (2013). The role of elementary mathematics specialists in the teaching and learning of mathematics. AMTE website: https://amte.net/sites/default/files/emspo sitionstatement_amte.pdf.

Baker, C., \& Knapp, M. (2019). The decision-making protocol for mathematics coaching: Addressing the complexity of coaching with intentionality and reflection. Mathematics Teacher Educator, 7(2), $27-43$.

Baker, C. K., Bitto, L., Wills, T., Galanti, T. M., \& Eatmon, C. (2018). Developing teacher leaders through self-study: A mathematics education field experience. In T. E. Hodges \& A. Baum (Eds.), Handbook of Research on Teacher Education (pp. 635-658). IGI Global.

Ball, D. L., Thames, M. H., \& Phelps, G. (2008). Content knowledge for teaching: What makes it special? Journal of Teacher Education, 59(5), 389-407. https://doi.org/10.1177/0022487108324554

Beswick, K. (2012). Teachers' beliefs about school mathematics and mathematicians' mathematics and their relationship to practice. Educational Studies in Mathematics, 79, 127-147. https://doi.org/10.1007/ s10649-011-9333-2

Brahier, D. J. (2000). Teaching secondary and middle school mathematics. Allyn and Bacon.

Bryk, A. S., Sebring, P. B., Allensworth, E., Luppescu, S., \& Easton, J. Q. (2010). Organizing schools for improvement: Lessons from Chicago. University of Chicago Press.

Campbell, C., Ellington, A., Haver, W., \& Inge, V. (2013). The elementary mathematics specialist handbook. National Council of Teachers of Mathematics.

Campbell, P., \& Malkus, N. (2011). The impact of elementary mathematics coaches on student achievement. The Elementary School Journal, 111, 430-454.

Campbell, P., \& Malkus, N. (2014). The mathematical knowledge and beliefs of elementary mathematics specialist-coaches. ZDM, 46, 213-222.

Chval, K. B., Arbaugh, F., Lannin, J. K., van Garderen, D., Cummings, L., Estapa, A. T., \& Huey, M. E. (2010). The transition from experienced teacher to mathematics coach: establishing a new identity. The Elementary School Journal, 111(1), 191-216. https://doi.org/10.1086/653475 
Clarke, S., Timperley, H., \& Hattie, J. (2004). Unlocking formative assessment: Practical strategies for enhancing students' learning in the primary and intermediate classroom. Hodder Moa Beckett.

Coburn, C. E., \& Russell, J. L. (2008). District policy and teachers' social networks. Educational Education and Policy Analysis, 30, 203-235.

Cooney, T. J. (1999). Conceptualizing teachers' ways of knowing. Educational Studies in Mathematics, $38,163-187$.

National Council of Teachers of Mathematics. (2014). Principles to actions: Ensuring mathematical success for all. NCTM.

Council of Chief State School Officers. (2013). Interstate Teacher Assessment and Support Consortium. In TASC Model Core Teaching Standards and Learning Progressions for Teachers 1.0: A Resource for Ongoing Teacher Development. Author.

Darling-Hammond, L., Hyler, M. E., Gardner, M. (2017). Effective Teacher Professional Development. Learning Policy Institute.

Daro, P, D., Mosher, F. A., \& Corcoran, T. (2011). Learning trajectories in mathematics: A foundation for standards, curriculum, assessment, and instruction. Consortium for Policy Research in Education.

Elementary Mathematics Specialists \& Teachers Leaders Project. (2019). Mathematics Specialist Certification by State http://www.mathspecialists.org/state-certifications.html.

Fennell, F. (2017). We need mathematics specialists now: A historical perspective and next steps. In M. McGatha \& N. Rigelman (Eds.), Elementary mathematics specialists (pp. 3-18). Association of Mathematics Teacher Educators.

Fennell, F., Kobett, B. M., \& Wray, J. A. (2013). Elementary mathematics leaders. Teaching Children Mathematics, 20(3), 172-180.

Gamoran, A. (2003). Access to resources. In A. Gamoran, C. W. Anderson, P. A. Quiroz, W. G. Secada, T. Williams, \& S. Ashmann (Eds.), Transforming teaching in math and science: How schools and districts can support change (pp. 65-86). Teachers College Press.

Generating accounts of mathematics teachers' practice. Journal for Research in Mathematics Education, $30,252-264$.

Gibbons, L. K., \& Cobb, P. (2017). Focusing on teacher opportunities to identify potentially productive coaching activities. Journal of Teacher Education, 68(4), 411-425. https://doi.org/10.1177/00224 87117702579

Grossman, P., Hammerness, K., \& McDonald, M. (2009). Redefining teaching, re-imagining teacher education. Teachers and Teaching: Theory and Practice, 12(2), 273-289. https://doi.org/10.1080/ 13540600902875340

Guba, E. G., \& Lincoln, Y. S. (1989). Fourth generation evaluation. Sage.

Guskey, T. R. (1986). Staff development and the process of teacher change. Educational Researcher, 15(4), 5-12.

Guskey, T. R. (2000). Evaluating professional development. Corwin Press.

Hiebert, J., Morris, A. K., Berk, D., \& Jansen, A. (2007). Preparing teachers to learn from teaching. Journal of Teacher Education, 58, 47-61.

Hjalmarson, M. A. (2017). Learning to teach math specialists in a synchronous online course: a selfstudy. Journal for Mathematics Teacher Education., 20(3), 281-301. https://doi.org/10.1007/ s10857-015-9323-x

Houston, W. R., \& Pugach, M. C. (Eds.), Encouraging reflective practice in education: An analysis of issues and programs 186-207. Teachers College Press.

Huinker, D. (2013). Dimensions of fraction operation sense. In Defining mathematics education: Presidential yearbook selections 1926-2012, seventy-fifth yearbook of the national council of teachers of mathematics (NCTM), (pp. 373-380). National Council Teachers of Mathematics.

Knapp, M. C. (2017). An autoethnography of a (reluctant) teacher leader. The Journal of Mathematical Behavior, 46, 251-266. https://doi.org/10.1016/j.jmathb.2017.02.004

Lampert, M. (2001). Teaching problems and the problems of teaching. Yale University Press.

Lesseig, K., Elliott, R., Kazemi, E., Kelley-Petersen, M., Campbell, M., Mumme, J., \& Carroll, C. (2017). Leader noticing of facilitation in videocases of mathematics professional development. Journal of Mathematics Teacher Education, 20, 591-619. https://doi.org/10.1007/s10857-016-9346-y

Lloyd, G. (2002). Mathematics teachers' beliefs and experiences with innovative curriculum materials. In G. Leder, E. Pehkonen, \& G. Toerner (Eds.), Beliefs: A hidden variable in mathematics education (pp. 1-11). Kluwer.

Loucks-Horsley, S., Stiles, K. E., Mundry, S., Love, N., \& Hewson, P. W. (2010). Designing professional development for teachers of science and mathematics. Corwin Press, 
Marzano, R. J. (2003). What works in schools: Translating research into action. Association of Supervision and Curriculum Development.

Maxwell, J. A. (2005). Qualitative research design: An interactive approach. (2nd ed.) Sage.

McGatha, M. B. \& Rigelman, N. R. (2017). Introduction. In M. McGatha \& N. Rigelman (Eds.), Elementary mathematics specialists (pp. xiii-Xv). Association of Mathematics Teacher Educators.

McTighe, J., \& Wiggins, G. P. (2013). Essential questions: Opening doors to student understanding. Association for Supervision and Curriculum Development.

National Council of Teachers of Mathematics. (2000). Principles and standards for school mathematics. NCTM.

National Council of Teachers of Mathematics. (2012). NCTM CAEP standards - elementary mathematics specialist. NCTM website: http://www.nctm.org/Standards-and-Positions/CAEP-Standards/.

National Mathematics Advisory Panel. (2008). Foundations for success: Final report of the National Mathematics Advisory Panel. U.S. Department of Education.

National Research Council (NRC). (2001). Adding it up: Helping children learn mathematics. In J. Kilpatrick, J. Swafford, \& B. Findell (Eds.), Mathematics learning study committee, center for education, division of behavioral and social sciences and education. Washington, DC: National Academy Press.

National Research Council. (2009). Mathematics learning in early childhood: Paths toward excellence and equity. In Christopher T. Cross, Taniesha A. Woods, and Heidi Schweingruber, (Eds), Committee on early childhood mathematics, center for education, division of behavioral and social sciences and education. National Academies Press.

Nelson, B. S. (1998). Lenses on learning: Administrators' views on reform and the professional development of teachers. Journal of Mathematics Teacher Education, 1, 191-215.

Patton, M. Q. (2002). Qualitative Research and evaluation methods (3rd ed.). Sage.

Phillips, R. A. (2007). Mathematics teachers' beliefs and affect. In F. Lester Jr. (Ed.), Second handbook of research on mathematics teaching and learning: A project of the national council of teachers of mathematics (pp. 257-315). Information Age Publishing Inc.

Poglinco, S. M., Bach, A. J., Hovde, K., Rosenblum, S., Saunders, M., \& Supovitz, J. A. (2003). The heart of the matter: The coaching model in America's choice schools. Consortium for Policy Research in Education, University of Pennsylvania.

Polly, D., Algozzine, R., \& Mraz, M. (2013). Implications for developing and researching elementary school mathematics coaches. School Science and Mathematics, 113(6), 297-307.

Pugach, M. C., \& Johnson, L. J. (1990). Developing reflective practice through structured dialogue. In R. T. Clift.

Resnick, L. B. (2010). Nested learning systems for the thinking curriculum. Educational Researcher, 39, $183-197$.

Saldãna, J. (2016). The coding manual for qualitative researchers. Sage.

Schoenfeld, A. (1988). When good teaching leads to bad results: The disasters of "well taught" mathematics courses. Educational Psychologist, 23, 145-166.

Schön, D. A. (1983). The reflective practitioner: How professionals think in action. Basic Books.

Shadish, W. R. (1995). Philosophy and the quantitative-qualitative debates: Thirteen common errors. Evaluation and Program Planning, 18(1), 63-75.

Showers, B. (1985). Teachers coaching teachers. Educational Leadership, 42(7), 43-49.

Simon, M. A., \& Tzur, R. (1999). Explicating the teacher's perspective from the researchers' perspectives: Generating accounts of mathematics teachers' practice. Journal for Research in Mathematics Education, 30, 252-264.

Smith, J. P. (1996). Efficacy and teaching mathematics by telling: a challenge for reform. Journal for Research in Mathematics Education, 27, 387-402.

Sowder, J. T. (2007). The mathematical education and development of teachers. In F. Lester Jr. (Ed.), Second handbook of research on mathematics teaching and learning: A project of the national council of teachers of mathematics (pp. 157-224). Information Age Publishing Inc.

Stake, R. E. (1995). The art of case study research. Sage.

Stake, R. E. (2006). Multiple case study analysis. The Guilford Press.

Stein, M. K., Smith, M. S., \& Silver, E. A. (1999). The development of professional developers: learning to assist teachers in new settings in new ways. Harvard Educational Review, 69, 237-269.

Stigler, J. W., \& Hiebert, J. (1999). The teaching gap: Best ideas from the world's teachers for improving education in the classroom. The Free Press.

Stylianou, D. A., \& Silver, E. A. (2004). The role of visual representations in advanced mathematical problem solving: an examination of expert-novice similarities and differences. Mathematical Thinking and Learning, 6(4), 353-387. 
Swars, S. L., Smith, S. Z., Smith, M. E., Carothers, J., \& Myers, K. (2018). The preparation experiences of elementary mathematics specialists examining influences beliefs, content knowledge, and teaching practices. Journal of Mathematics Teacher Education, 21(2), 123-145. https://doi.org/10.1007/ s10857-016-9354-y

Sykes, G. (1999). Teacher and student learning: Strengthening the connection. In L. Darling-Hammond \& G. Sykes (Eds.), Teaching as the learning profession: Handbook of policy and practice (pp. 151-179). Jossey-Bass.

Sztajn, P., Confrey, J., Wilson, P. H., \& Edgington, C. (2012). Learning trajectory based instruction: toward a theory of teaching. Educational Researcher, 41(5), 147-156.

Trotter, Y. D. (2006). Adult learning theories: Impacting professional development programs. Delta Kappa Gamma Bulletin, 72(2), 8.

Warfield, J. (2001). Where mathematics content matters: Learning about and building on children's mathematical thinking. In T. Wood, B. S. Nelson, \& J. Warfield (Eds.), Beyond classical pedagogy: Teaching elementary school mathematics (pp. 135-155). Erlbaum.

Weiss, H. B. \& Greene, G. C. (1992). An empowering partnership for family support and education programs and evaluations. Family Science Review, 5(1, 2, February/May), 145-163.

Yin, R. K. (2009). Case study research: Design and methods. (4th ed.). Sage.

Zimmerman, B. J. (2001). Theories of self-regulated learning and academic achievement: An overview and analysis. In B. J. Zimmerman \& D. H. Schunk (Eds.), Self-regulated learning and academic achievement: Theoretical perspectives (pp. 1-65). Erlbaum.

Publisher's Note Springer Nature remains neutral with regard to jurisdictional claims in published maps and institutional affiliations. 